Original paper

\title{
Ammonia-induced mitochondrial dysfunction and energy metabolism disturbances in isolated brain and liver mitochondria, and the effect of taurine administration: relevance to hepatic encephalopathy treatment
}

\author{
Hossein Niknahad', Akram Jamshidzadeh'1, Reza Heidari', Mahdi Zarei', Mohammad Mehdi Ommati \\ 'Pharmaceutical Sciences Research Center, Shiraz University of Medical Sciences, Shiraz, Iran \\ 2Department of Animal Sciences, School of Agriculture, Shiraz University, Shiraz, Iran
}

\begin{abstract}
Introduction: Ammonia-induced oxidative stress, mitochondrial dysfunction, and energy crisis are known as some the major mechanisms of brain injury in hepatic encephalopathy (HE). Hyperammonemia also affects the liver and hepatocytes. Therefore, targeting mitochondria seems to be a therapeutic point of intervention in the treatment of $\mathrm{HE}$. Taurine is an abundant amino acid in the human body. Several biological functions including the mitochondrial protective properties are attributed to this amino acid. The aim of this study is to evaluate the effect of taurine administration on ammonia-induced mitochondrial dysfunction.

Material and methods: Isolated mice liver and brain mitochondria were exposed to different concentrations of ammonia $(1,5,10$, and $20 \mathrm{mM})$ and taurine $(1,5$, and $10 \mathrm{mM})$, and several mitochondrial indices were assessed.

Results: It was found that ammonia inhibited mitochondrial dehydrogenases activity caused collapse of mitochondrial membrane potential (MMP), induced mitochondrial swelling (MPP), and increased reactive oxygen species (ROS) in isolated liver and brain mitochondria. Furthermore, a significant amount of lipid peroxidation (LPO), along with glutathione (GSH) and ATP depletion, was detected in ammonia exposed mitochondria. Taurine administration (5 and $10 \mathrm{mM}$ ) mitigated ammonia-induced mitochondrial dysfunction.

Conclusions: The current investigation demonstrates that taurine is instrumental in preserving brain and liver mitochondrial function in a hyperammonemic environment. The data suggest taurine as a potential protective agent with a therapeutic capability against hepatic encephalopathy and hyperammonemia.
\end{abstract}

Key words: amino acid, bioenergetics, brain injury, hepatic encephalopathy, taurine.

Address for correspondence

Reza Heidari, Shiraz University of Medical Sciences, P. 0. Box 1583; 71345. Roknabad, Karafarin St., P. O. Box 1583; 71345 Shiraz, Fars, Iran, phone: +98 9171237882, e-mail: rezaheidari@hotmail.com

\section{Introduction}

Hepatic encephalopathy (HE) is a deleterious clinical complication accompanied by acute and chronic liver injury [1]. Although the exact cause of HE is unknown, there is agreement on the predominant role played by ammonia in HE etiology [2]. Ammonia is metabolized by the liver to the urea in healthy subjects. Damaged livers are unable to metabolize ammonia.
Hence, this toxic chemical is elevated in the systemic circulation and finally reaches the brain. Ammonia is a neurotoxin that mainly affects astrocytes in the central nervous system (CNS) [3, 4]. It also has several direct toxic effects on neurons [4]. Ammonia causes brain edema, oxidative stress, and inflammation when its level rises in HE [5]. Consequently, a decline in brain function occurs in patients with HE [5]. Hyperammonemia also affects hepatocytes [6]. 
Disturbed mitochondrial function and oxidative stress are implicated in ammonia-induced cytotoxicity $[3,7]$. It has been reported that brain energy metabolism is disturbed in chronic and acute HE [8-10]. Ammonia negatively affects several key enzymes that are responsible for energy metabolism in mitochondria [11]. Hence, the targeting of bioenergetics failure represents a potentially useful approach for the treatment of HE and hyperammonemia disorders.

Taurine is an abundant non-protein amino acid in the human body. The concentrations of taurine in the brain, heart, and muscle are high [12]. Several physiological and pharmacological roles are attributed to taurine [13]. This amino acid is an antioxidant, membrane stabilizer, osmoregulator, and most probably, a neurotransmitter [14-16]. On the other hand, the protective effect of taurine has been investigated against a range of different xenobiotics [17-20]. Taurine has also been shown to have a profound effect on CNS [21, 22]. It has been shown that taurine acts as an osmoregulator, protects neurons, prevents astrocytes swelling, and encounters oxidative stress in CNS [23-26]. The protective effects of taurine against a range of CNS disorders, as well as xenobiotics-induced CNS injury have also been reported [21-26].

The effect of taurine on mitochondria is reported in several investigations $[14,27,28]$. Taurine regulates mitochondrial $\mathrm{pH}$, affects mitochondrial GSH and antioxidants, and preserves the mitochondrial membrane potential $[14,29,30]$.

Recently, we found that taurine administration effectively prevented the increase in plasma and brain ammonia after chronic and acute liver failure [31]. The aim of this study is to evaluate the effect of taurine on brain and liver mitochondria as a major target of ammonia-induced toxicity in $\mathrm{HE}$.

\section{Material and methods}

\section{Chemicals}

4,2-Hydroxyethyl, 1-piperazineethanesulfonicacid (HEPES), 6-hydroxy-2,5,7,8-tetramethylchroman-2-carbo2-carboxylic acid (Trolox), 3-(N-morpholino) propane sulfonic acid (MOPS), dimethyl sulfoxide (DMSO), D-mannitol, fatty acid-free bovine serum albumin (BSA) fraction V, Thiobarbituric acid (TBA), 3-[4, 5 dimethylthiazol-2-yl]-2, 5-diphenyltetrazolium bromide (MTT), Dithiobis-2-nitrobenzoic acid (DTNB), Glutathione (GSH), 2', 7'-Dichlorofluorescein diacetate (DCFH-DA), Taurine, Malondialdehyde (MDA), Sucrose, $\mathrm{KCl}, \mathrm{Na}_{2} \mathrm{HPO}_{4}, \mathrm{MgCl}_{2}$, Rhodamine 123 (Rh-123), Coomassie brilliant blue, Ethylene glycol-bis (2-amino-
ethylether)-N,N,N',N'-tetraacetic acid (EGTA), Sodium succinate, and Ethylenediaminetetraacetic acid (EDTA) were purchased from Sigma Chemical Co. (St. Louis, MO, USA). Trichloroacetic acids (TCA), ammonium chloride, and hydroxymethyl amino methane hydrochloride (Tris-HCl) were purchased from Merck (Dardamstd, Germany). All salts for preparing buffer solutions were of analytical grade and prepared from Merck (Dardamstd, Germany).

\section{Animals}

Male BALB/c mice ( $n=60,25-30 \mathrm{~g})$ were housed in cages on wood bedding at a temperature of $23 \pm 1^{\circ} \mathrm{C}$. Animals had free access to food and tap water. The animals received humane care and were handled according to the animal handling protocol approved by a local ethics committee (\# 94-01-36-9741).

\section{Mitochondria isolation}

Mice liver and brain mitochondria were isolated as previously described [32]. Briefly, animals were anesthetized (Thiopental, $50 \mathrm{mg} / \mathrm{kg}$, i.p.), and their brain and liver were excised, washed and minced in icecooled saline (sodium chloride $0.9 \%$ ) [32,33]. Mice liver and brain was homogenized in an ice-cooled buffer containing $75 \mathrm{mM}$ mannitol, $220 \mathrm{mM}$ sucrose, $0.5 \mathrm{mM}$ EGTA, $2 \mathrm{mM}$ HEPES, $0.1 \% \mathrm{BSA}(\mathrm{pH}=7.4)$ at a $10: 1$ buffer to tissue $(\mathrm{v} / \mathrm{w})$ ratio [32]. Afterward, tissue homogenate was centrifuged at $1,000 \times \mathrm{g}$ for 10 minutes at $4^{\circ} \mathrm{C}$ to remove intact cell debris and nuclei. The supernatants were further centrifuged at $10,000 \times \mathrm{g}\left(4^{\circ} \mathrm{C}\right.$ for 10 minutes) to precipitate the heavy membrane fractions (mitochondria). The recent step was repeated three times using fresh buffer medium. As mentioned, all manipulations for mitochondria isolation were performed at $4^{\circ} \mathrm{C}$ or on ice to minimize mitochondrial injury [32].

Ammonia was added with previously reported pathologically relevant concentrations $(5 \mathrm{mM}, 10 \mathrm{mM})$ and with a high concentration $(20 \mathrm{mM})$, to the isolated brain and liver mitochondria, and the toxicity markers were monitored [34].

\section{Mitochondrial dehydrogenases activity}

The 3-(4, 5-dimethylthiazol-2-yl)-2, 5-diphenyltetrazolium bromide (MTT) assay was applied as a colorimetric method for determination of mitochondrial functionality in isolated mitochondria [35, 36]. Briefly, mitochondrial suspension in a buffer containing $320 \mathrm{mM}$ sucrose, $1 \mathrm{mM}$ EDTA, and $10 \mathrm{mM}$ Tris- $\mathrm{HCl}$ 
( $\mathrm{pH}=7.4)$, was incubated with $0.4 \%$ of $\mathrm{MTT}$ at $37^{\circ} \mathrm{C}$ for 30 minutes. The product of purple formazan crystals was precipitated $(10,000 \mathrm{~g}, 10 \mathrm{~min})$ and dissolved in $1 \mathrm{~mL}$ dimethyl sulfoxide (DMSO). Then, $100 \mu \mathrm{l}$ of dissolved formazan was added to 96 well plate and the optical density (OD) at $570 \mathrm{~nm}$ was measured with an EPOCH plate reader (BioTek ${ }^{\circ}$ Instruments, Highland Park, USA).

\section{Reactive oxygen species (ROS) in isolated mitochondria}

The mitochondrial ROS measurement was performed using the fluorescent probe DCFH-DA [32]. Briefly, isolated kidney mitochondria were placed in a buffer containing $125 \mathrm{mM}$ sucrose, $65 \mathrm{mM} \mathrm{KCl}$, $10 \mathrm{mM}$ HEPES, $5 \mathrm{mM}$ Sodium succinate, and $20 \mu \mathrm{M}$ $\mathrm{Ca}^{2+}(\mathrm{pH}=7.2)$ [32]. Following this step, DCFH-DA was added (final concentration, $10 \mu \mathrm{M}$ ) to mitochondrial suspension, and then incubated for $30 \mathrm{~min}$ at $37^{\circ} \mathrm{C}$. The fluorescence intensity of the samples was measured using a FLUOstar Omega ${ }^{\circ}$ multifunctional microplate reader $\left(\lambda_{\text {excitation }}=485 \mathrm{~nm}\right.$ and $\lambda_{\text {emission }}=$ $=525 \mathrm{~nm}$ ) [32].

\section{Mitochondrial membrane potential}

Mitochondrial uptake of the cationic fluorescent dye, rhodamine 123, has been used for the estimation of mitochondrial membrane potential [32]. For this purpose, the mitochondrial fractions $(0.5 \mathrm{mg}$ protein/ $\mathrm{ml})$ were incubated $\left(30 \mathrm{~min}, 37^{\circ} \mathrm{C}\right)$ with $10 \mu \mathrm{M}$ of rhodamine 123 in a buffer containing $125 \mathrm{mM}$ sucrose, $65 \mathrm{mM} \mathrm{KCl}, 10 \mathrm{mM}$ HEPES, $5 \mathrm{mM}$ Sodium succinate, and $20 \mu \mathrm{M} \mathrm{Ca}^{2+}(\mathrm{pH}=7.2)$. Samples were centrifuged $\left(10 \mathrm{~min}, 10,000 \mathrm{~g}, 4^{\circ} \mathrm{C}\right)$, and the fluorescence intensity of supernatant was monitored using a FLUOstar $\mathrm{Omega}^{\circ}$ multifunctional microplate reader at the excitation and emission wavelength of $485 \mathrm{~nm}$ and $525 \mathrm{~nm}$, respectively [32].

\section{Mitochondrial swelling assay}

Mitochondrial swelling was measured as previously described [32]. Briefly, the mitochondria were suspended in a buffer containing $125 \mathrm{mM}$ sucrose, $65 \mathrm{mM}$ $\mathrm{KCl}, 10 \mathrm{mM}$ Hepes-KOH, $20 \mu \mathrm{M} \mathrm{Ca}^{2+}(\mathrm{pH}=7.2)$. Light absorbance at $540 \mathrm{~nm}$ was measured at the twotime points (10 and 70 minutes) at $30^{\circ} \mathrm{C}$ [32]. It is accepted that a decreased light absorbance is consistent with an increase in mitochondrial volume. Hence, as mitochondria are more swelled, the differences between light absorbance of two-time points are high- er. The differences between the absorbance of samples were assessed $\left(\Delta \mathrm{OD}^{540 \mathrm{~nm}}\right)$ and compared in different experimental groups [32].

\section{Lipid peroxidation}

Thiobarbituric acid reactive substances (TBARS) were assessed as an index of lipid peroxidation in isolated liver and brain mitochondria [32]. Briefly, isolated mitochondria were washed to remove sucrose in ice-cold 3-(N-morpholino) propane sulfonic acid (MOPS)-KCl buffer (50 mM MOPS, $100 \mathrm{mM} \mathrm{KCl}$, $\mathrm{pH}=7.4$ ), and re-suspended in MOPS-KCl buffer. Afterward, the mitochondrial suspension was mixed with twice its volume of $15 \%$ trichloroacetic acid, $0.375 \%$ thiobarbituric acid (TBA), $0.24 \mathrm{~N} \mathrm{HCl}$ plus $0.5 \mathrm{mM}$ Trolox, and heated for $15 \mathrm{~min}$ at $100^{\circ} \mathrm{C}[32,37]$. After centrifugation $(15,000 \mathrm{~g}, 1 \mathrm{~min})$, the absorbance of the supernatant was assessed at $532 \mathrm{~nm}$ with an Epoch plate reader (BioTek ${ }^{\circledR}$ Instruments, Highland Park, USA) $[32,36]$.

\section{Mitochondrial glutathione (GSH) content}

GSH level was determined with the spectrophotometric method using 5, 50-dithiobis-2-nitrobenzoic acid (DTNB) as an indicator of GSH [32]. The mitochondrial suspension was treated with trichloroacetic acid $(10 \%, \mathrm{w} / \mathrm{v})$ to extract mitochondrial glutathione. The mixture was centrifuged $\left(15,000 \mathrm{~g}, 4^{\circ} \mathrm{C}, 1 \mathrm{~min}\right)$ to remove denatured proteins [32]. Afterward, $100 \mu \mathrm{l}$ of DTNB $(0.04 \%$ in phosphate buffer, $\mathrm{pH}=8.9)$ was added and the intensity of produced color was recorded at $412 \mathrm{~nm}$ with an ultraviolet spectrophotometer [36].

\section{Mitochondrial ATP level}

A luciferase-luciferin-based kit (ENLITEN ${ }^{\circledR}$, Promega) was used to assess mitochondrial ATP content [38]. Samples and buffer solutions were prepared based on the kit instructions and the luminescence intensity of samples was measured at $560 \mathrm{~nm}$ using a FLUOstar Omega multifunctional microplate reader.

\section{Samples protein content}

For standardization of data, samples protein concentrations were determined by the Bradford method [39].

\section{Statistical analysis}

Data are given as the mean \pm SD $(n=6)$. Data comparison was performed by the one-way analysis of 
variance (ANOVA) with Tukey's multiple comparison test as a post hoc. Differences were considered statistically significant when $p<0.05$.

\section{Results}

The alterations in mitochondrial dehydrogenases activity (MTT test) under the various incubation of $\mathrm{NH}_{4}^{+}$concentrations are summarized in Figure 1 . It was found that $\mathrm{NH}_{4}^{+}$concentrations higher than $5 \mathrm{mM}$ significantly decreased mitochondrial dehydrogenases activity in both isolated brain and liver mitochondria. Isolated mitochondria were also incubated with different taurine concentrations. Taurine caused no significant decrease in mitochondrial dehydrogenases activity when it was administered alone. Concentrations of $5 \mathrm{mM}$ and $10 \mathrm{mM}$ of taurine seem to preserve isolated mitochondria in a more functional state as judged by mitochondrial dehydrogenases activity (Fig. 1).
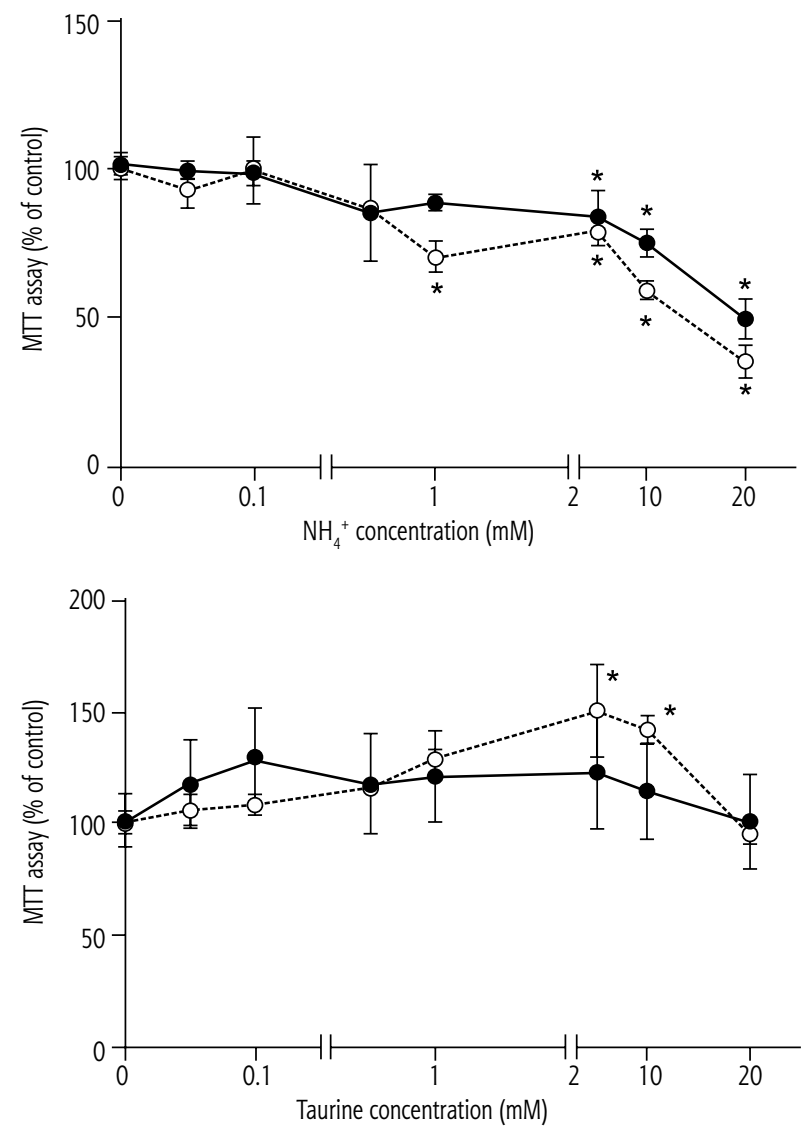

$\longrightarrow$ Brain mitochondria $\quad$-.-o--.. Liver mitochondria

Fig. 1. Concentration-response of ammonia and taurine in isolated mice brain and liver mitochondria. $\mathrm{NH}_{4}^{+}$: ammonium ion. Data are given as mean $\pm \mathrm{SD}$ $(n=6) .{ }^{*}$ - Indicates significantly different from concentration $0 \mathrm{mM}(p<0.05)$
The effect of taurine administration against ammoniainduced mitochondrial dysfunction was investigated. It was found that taurine $(5,10$, and $20 \mathrm{mM})$ significantly prevented the ammonia-induced decrease in mitochondrial dehydrogenases activity in isolated brain and liver mitochondria (Fig. 2).

A significant amount of reactive oxygen species (ROS) was detected in ammonia-treated groups (Fig. 3). On the other hand, taurine $(10 \mathrm{mM})$ effectively mitigated ammonia-induced oxidative stress in brain and liver mitochondria (Fig. 3).

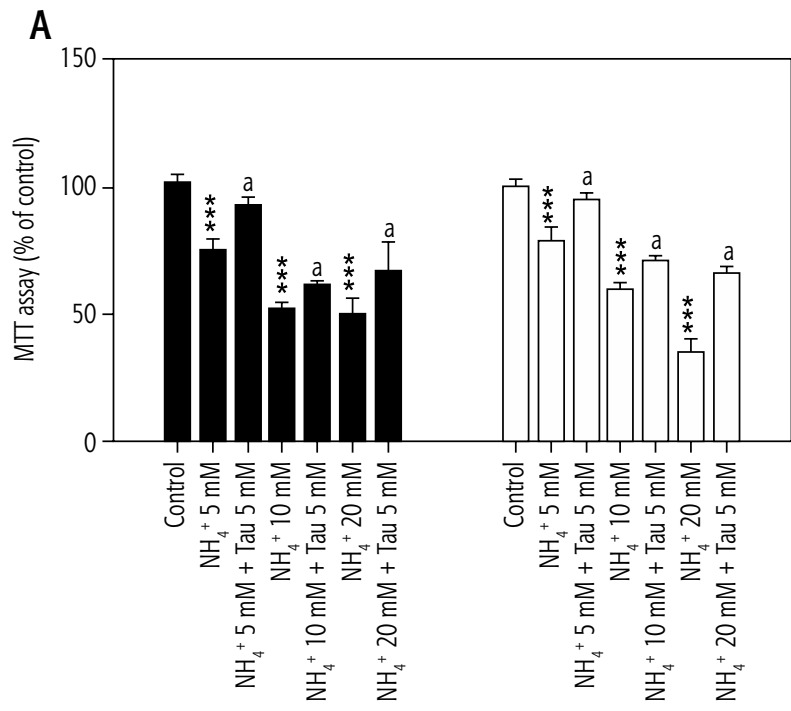

B

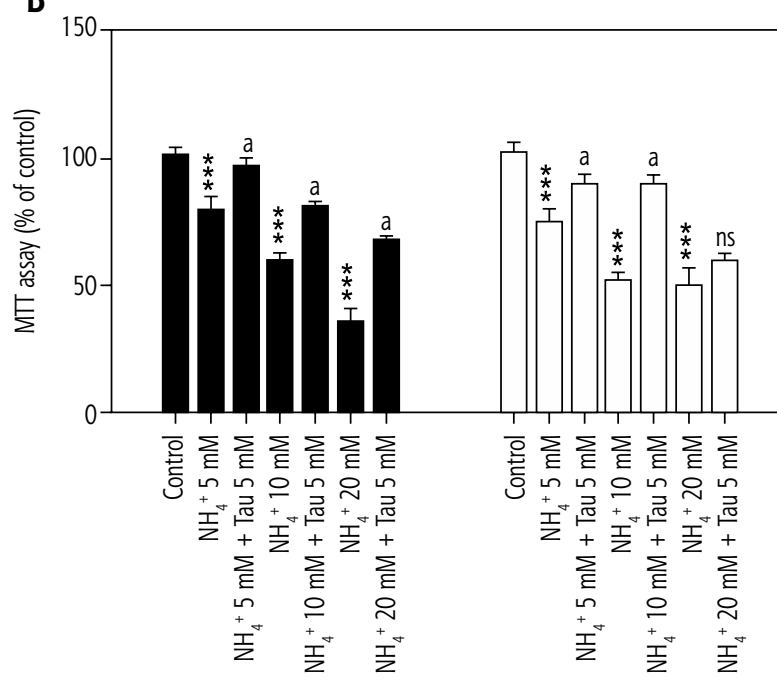

Brain mitochondria $\quad \square$ Liver mitochondria

Fig. 2. The effect of taurine administration on the ammonia-induced decrease in mitochondrial succinate dehydrogenase activity. A) Taurine $5 \mathrm{mM}$, B) Taurine $10 \mathrm{mM}$. Tau: taurine, $\mathrm{NH}_{4}^{+}$: ammonium ion. Values are represented as mean $\pm S D(n=6) .{ }^{* *}-$ Indicates significantly different from control $(p<0.001)$. a - Indicates significantly different from ammonia-treated group $(p<0.05)$. ns - Indicates not significant as compared with ammonia-treated group 

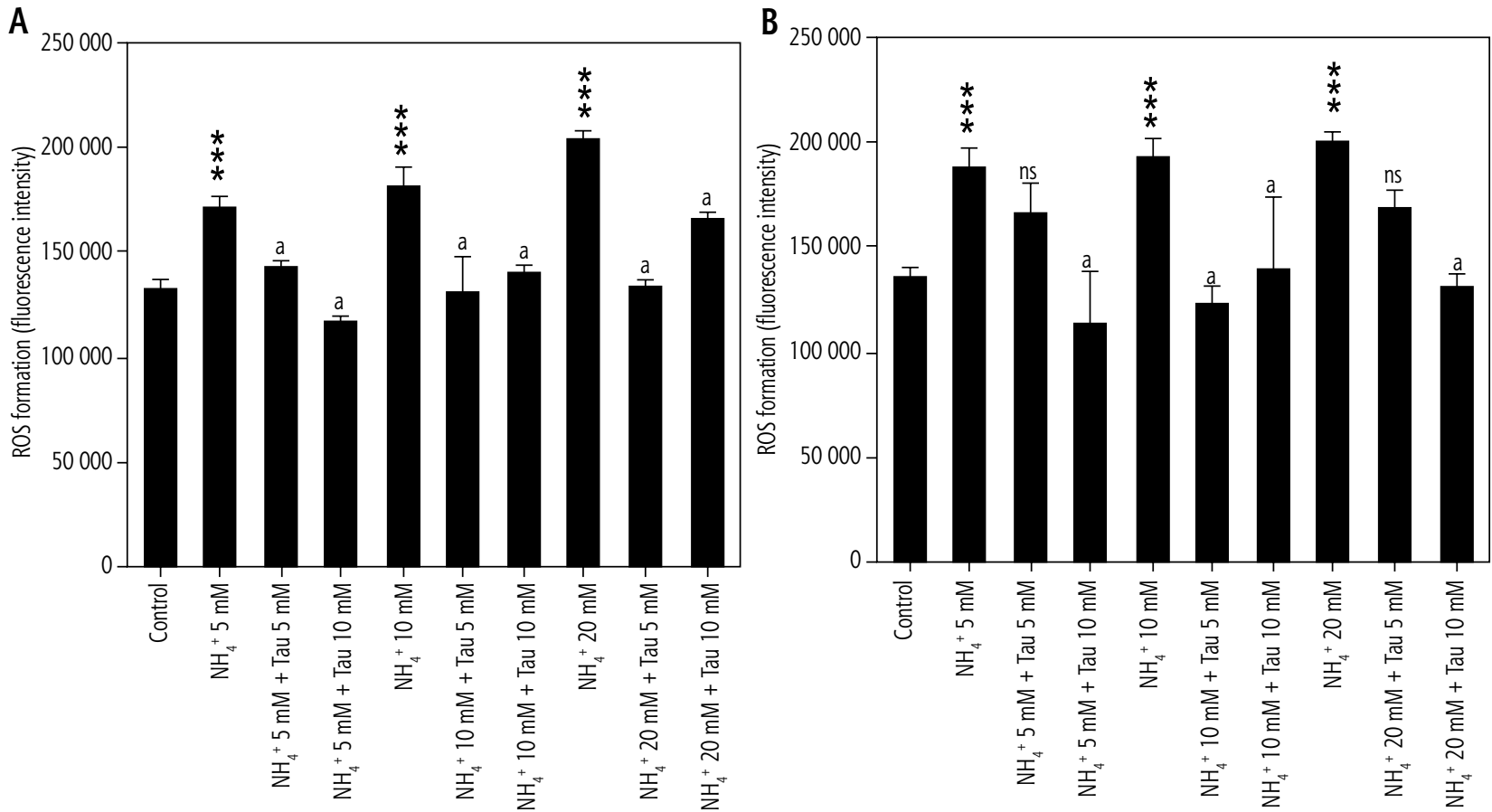

Fig. 3. Effect of taurine on ammonia-induced ROS formation in the isolated brain (A) and liver (B) mitochondria. Tau: taurine, $\mathrm{NH}_{4}^{+}$: ammonium ion. Data are given as mean $\pm S D(n=6) . * * *$ - Indicates significantly different from control $(p<0.001)$. a - Indicates significantly different from the only ammonium-treated group $(p<0.001)$. ns - not significant as compared with the ammonia-treated group
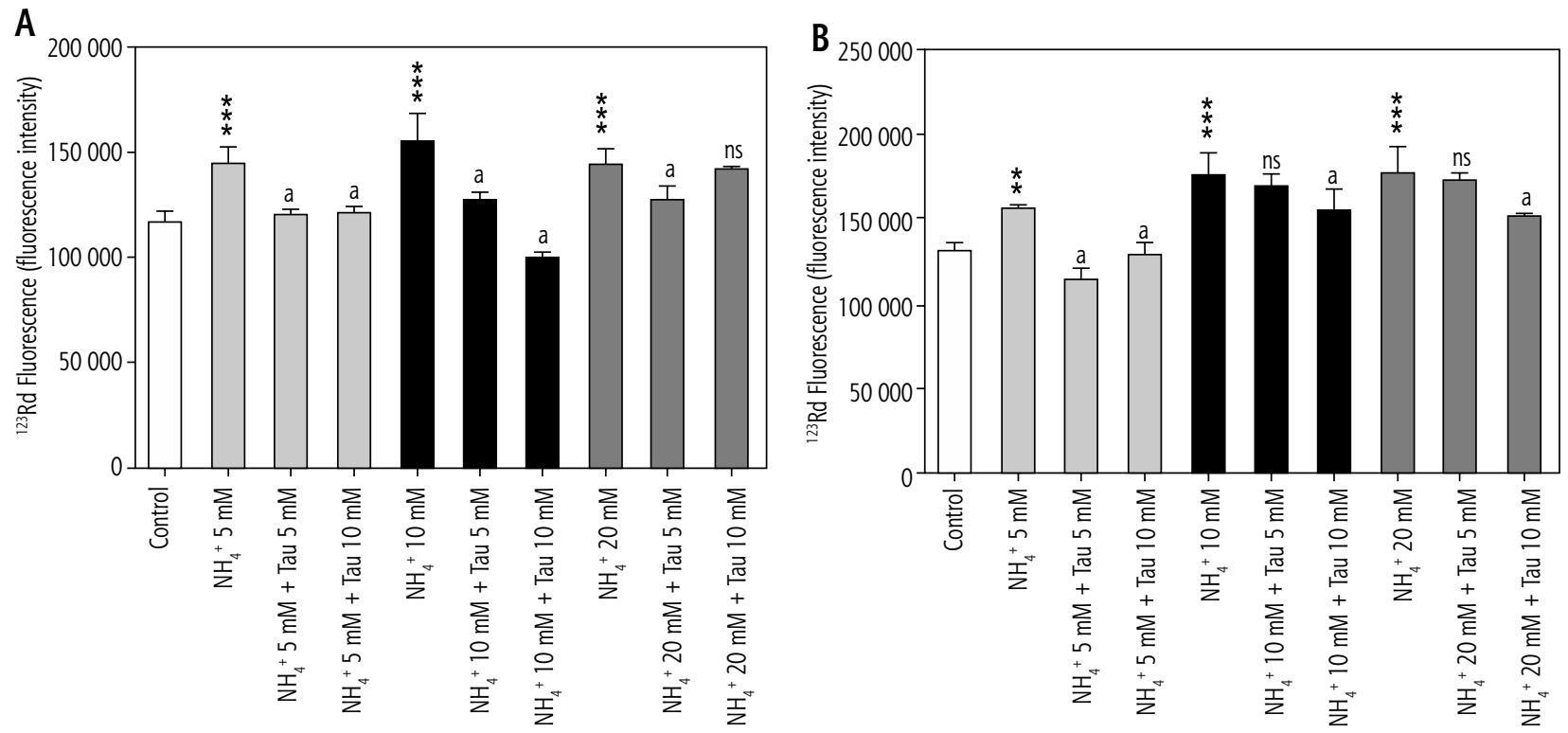

Fig. 4. Ammonia-induced mitochondrial membrane collapse and the effect of taurine. A) Isolated brain mitochondria, B) isolated liver mitochondria. Tau: taurine, $\mathrm{NH}_{4}^{+}$: ammonium ion. Asterisks indicate significantly different as compared with control $\left({ }^{* *} p<0.01,{ }^{* *} p<0.001\right)$. a - Indicates significantly different as compared to ammonia-treated group $(p<0.001)$. ns - Indicates not significant as compared with the ammonia-treated group

It was found that ammonia $(5,10$, and $20 \mathrm{mM})$ caused mitochondrial membrane potential $(\Delta \Psi)$ collapse (Fig. 4). Also, taurine $(10 \mathrm{mM})$, effectively mitigated the ammonia-induced decrease in mitochondrial membrane potential (Fig. 4).
When different concentrations of ammonia were added to isolated mitochondria, a marked increase of mitochondrial swelling was evident (Fig. 5). It was found that the induction by ammonia of mitochondrial swelling was significantly mitigated by taurine administration (Fig. 5). 

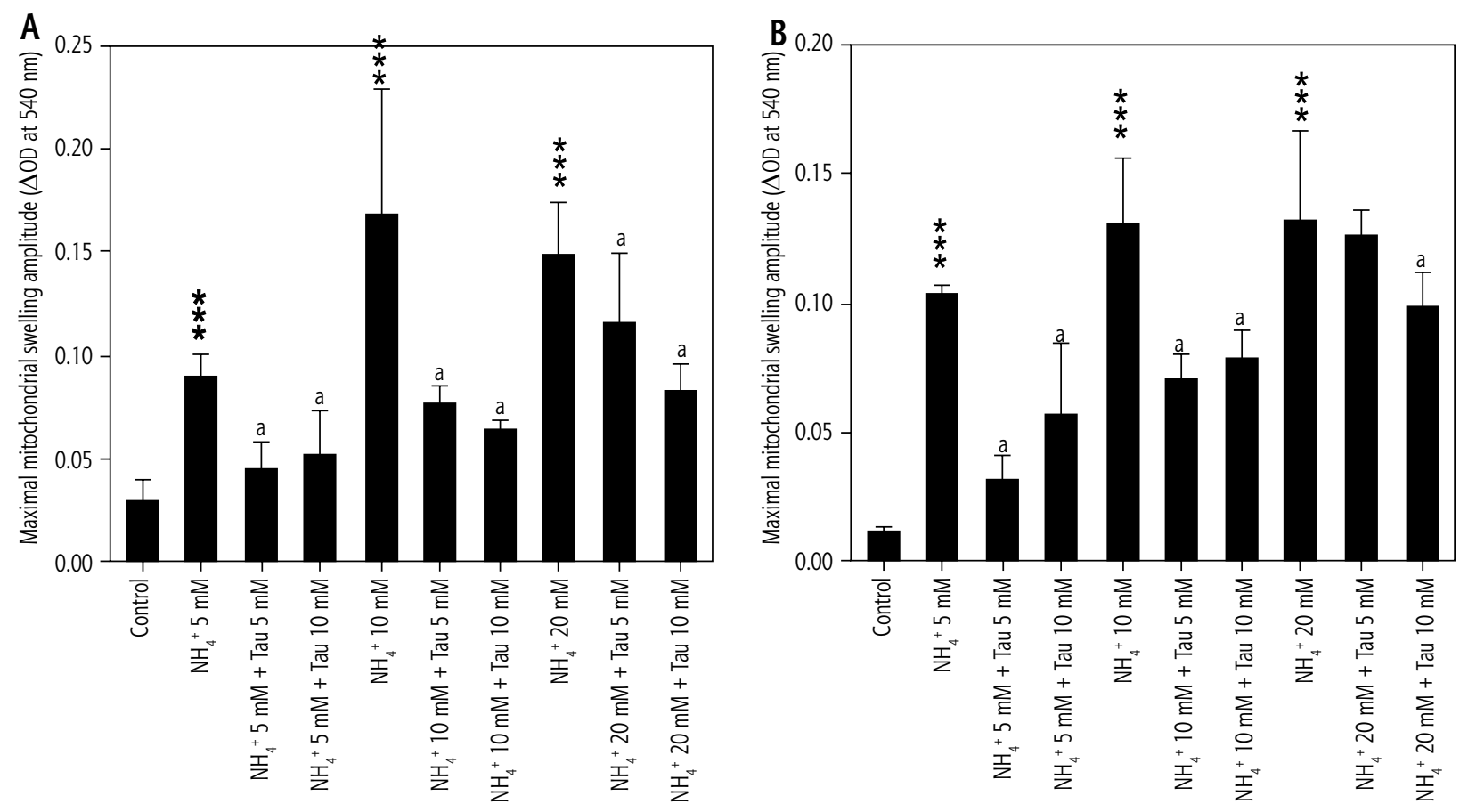

Fig. 5. Effect of taurine on ammonia-induced mitochondrial swelling. A) Isolated brain mitochondria, B) isolated liver mitochondria. Tau: taurine, $\mathrm{NH}_{4}^{+}$: ammonium ion. Data are represented as mean $\pm S D(n=6)$. ${ }^{* *}$ - Indicates significantly different from control $(p<0.001)$. a - Indicates significantly different as compared with the ammonia-treated group $(p<0.05)$. ns - not significant as compared with the ammonia-treated group
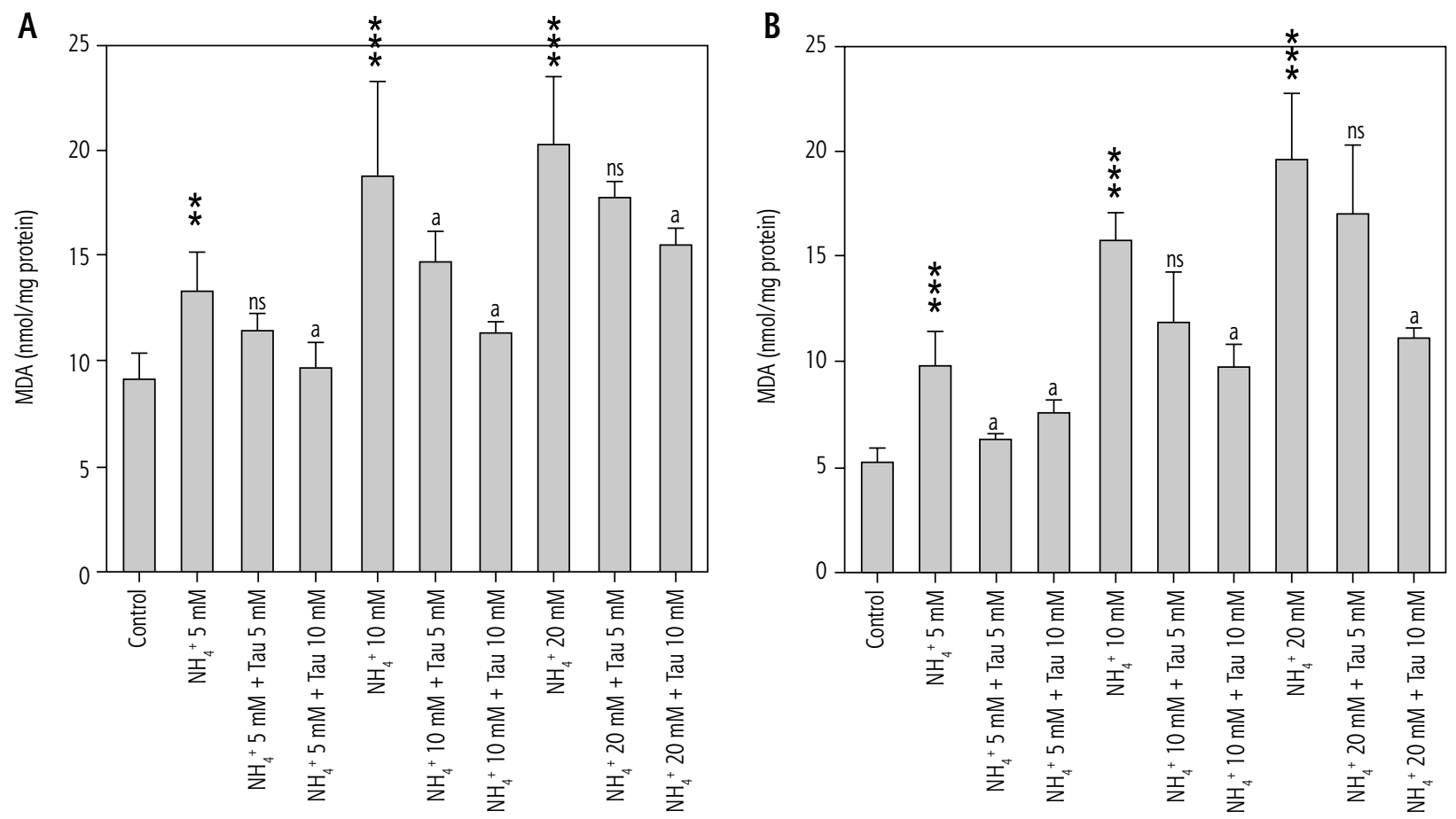

Fig. 6. Ammonia-induced lipid peroxidation in isolated brain (A) and liver (B) mitochondria. Tau: taurine, $\mathrm{NH}_{4}^{+}$: ammonium ion. Values are given as mean $\pm \mathrm{SD}$ $(n=6)$. Asterisks indicate significantly different as compared with control $\left({ }^{* *} p<0.01,{ }^{* *} p<0.001\right)$. a - Indicates significantly different as compared to the same ammonia concentration-treated group $(p<0.001)$. ns - not significant as compared with the ammonia-treated group 

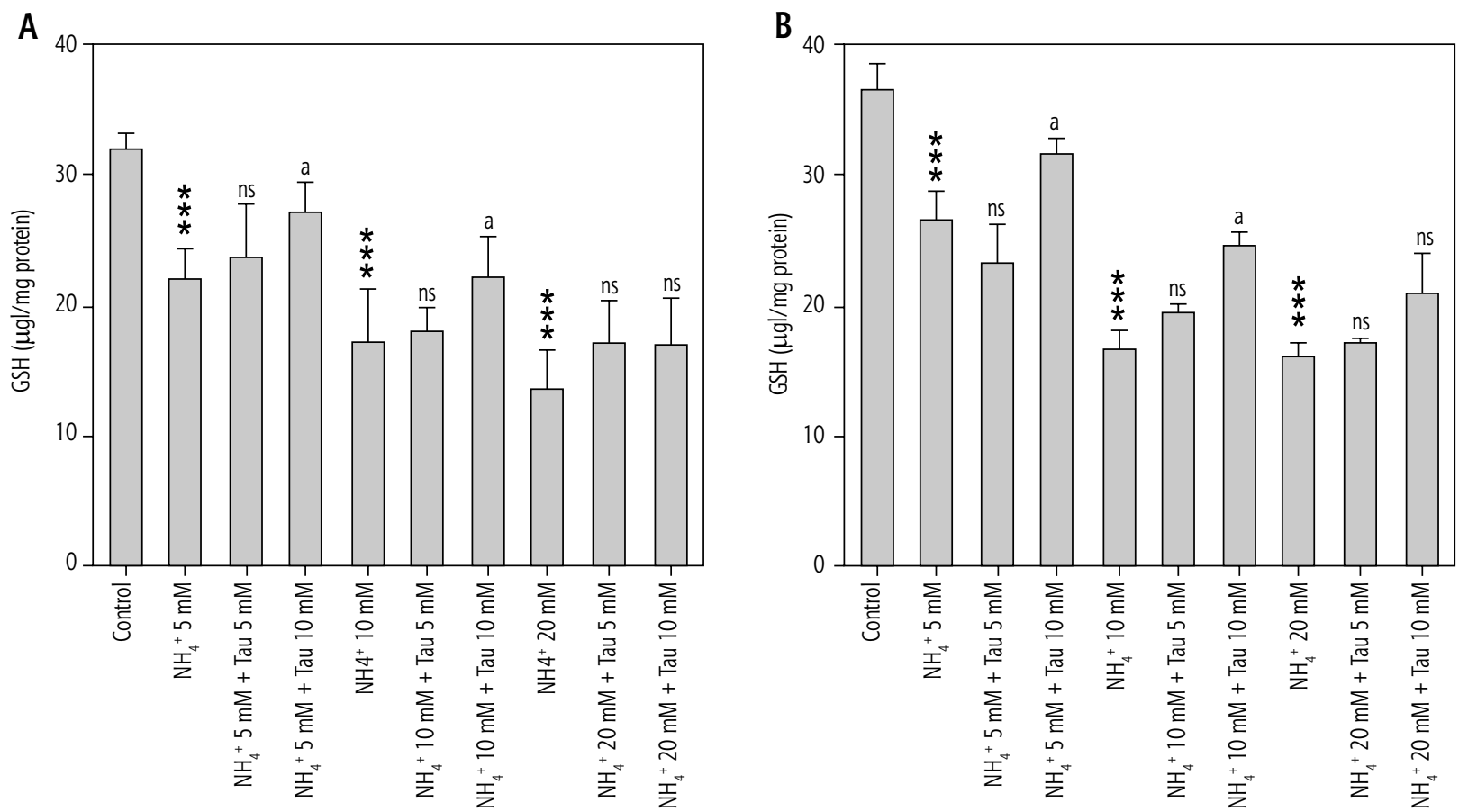

Fig. 7. The effect of ammonia on mitochondrial GSH content. A) Isolated brain mitochondria, B) isolated liver mitochondria. Tau: taurine, $\mathrm{NH}_{4}^{+}$: ammonium ion. Data are represented as mean $\pm S D(n=6)$. ${ }^{* *}$ - Indicates significantly different from control $(p<0.001)$. a - Indicates significantly different from the ammoniatreated group $(p<0.05)$. ns - not significant as compared with the ammonia-treated group
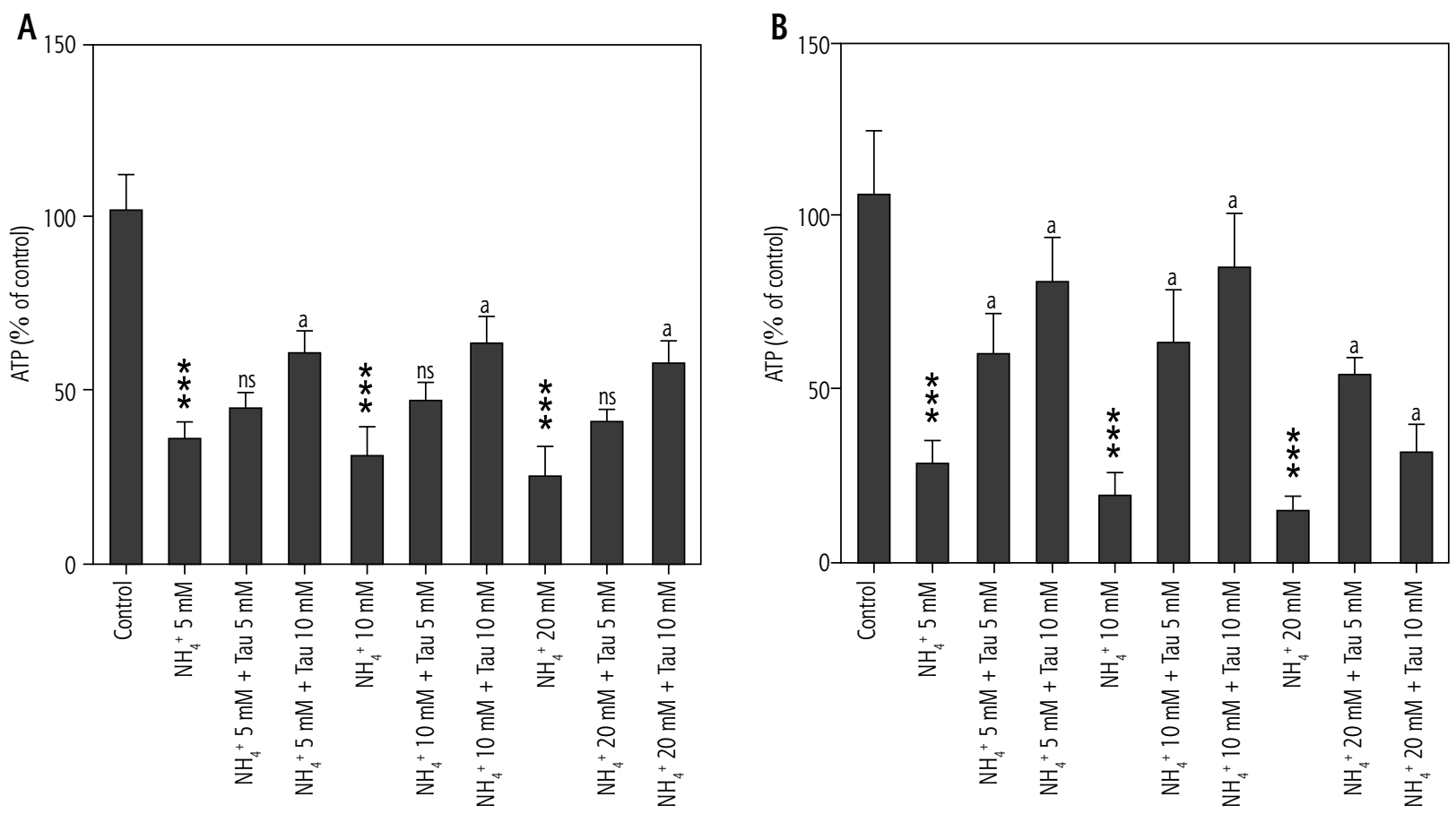

Fig. 8. Mitochondrial ATP level. Tau: taurine, $\mathrm{NH}_{4}^{+}$: ammonium ion. A) Isolated brain mitochondria, B) isolated liver mitochondria. Data are represented as mean \pm SD $(n=6) .{ }^{* * *}$ - Indicates significantly different from control $(p<0.001)$. a - Indicates significantly different from the ammonia-treated group $(p<0.05)$. ns - not significant as compared with the ammonia-treated group 
Ammonia also induced significant lipid peroxidation (Fig. 6) and glutathione and ATP depletion (Figs. 7 and 8). Taurine prevented ammonia-induced lipid peroxidation (Fig. 6), glutathione reservoir depletion (Fig. 7), and a decrease in mitochondrial ATP level (Fig. 8) in isolated brain and liver mitochondria.

\section{Discussion}

Ammonia is a neurotoxin underlying the pathogenesis of HE [2, 40]. Mitochondria are major targets of ammonia-induced cytotoxicity $[7,8,10]$. Hence, protecting this organelle may prevent ammonia-induced cellular injury and has clinical benefits in hyperammonemia conditions. In the current investigation, we found that taurine administration effectively preserved isolated brain and liver mitochondrial function in a hyperammonemic environment.

Ammonia-induced oxidative/nitrosative stress in CNS is accompanied by extensive lipid peroxidation and cellular glutathione reservoirs depletion [41, 42]. We found that ammonia caused significant lipid peroxidation and decrease in the glutathione content of the liver and brain mitochondria (Figs. 6 and 7). Taurine is a well-known biomembrane stabilizer and effectively mitigates oxidative stress and lipid peroxidation [13, 43]. This amino acid is also reported to preserve cellular glutathione reservoirs and boosts antioxidant defense mechanisms [44]. Hence, the antioxidant capacity of taurine could also be involved in its protective properties against ammonia-induced mitochondrial dysfunction. Interestingly, it has been found that the antioxidant properties of taurine might be mediated through the effect of this amino acid on mitochondria $[19,45]$.

High blood ammonia concentrations also affect hepatocytes and liver function [6]. Therefore, hyperammonemia might act as both: a cause and/or an effect of the hepatic injury. On the other hand, the beneficial effects of taurine on liver function and xenobiotics-induced liver injury have been extensively mentioned in previous investigations [17, 46-48]. Hence, taurine administration could be beneficial in HE and hyperammonemia by correcting liver function and preserving the liver capability for ammonia detoxification.

$\mathrm{Ca}^{2+}$ is a well-known inducer of mPT [49]. An ammonia-induced rise in $\mathrm{Ca}^{2+}$ levels has been found in cultured cells when exposed to this toxin [50]. Calcium chelators effectively mitigated ammonia-induced mitochondrial injury and cell swelling [50]. On the other side, taurine is a well-known agent, which potentiates the $\mathrm{Ca}^{2+}$ sequestering capability of mitochondria [27, 51]. Therefore, this unique property of this amino acid might be beneficial in mitigating ammonia-induced cytotoxicity.

Brain mitochondrial dysfunction in hepatic encephalopathy leads to disturbances in the cerebral energy metabolism [7, 8]. Ammonia affects several pathways, including the tricarboxylic acid cycle (TCA), lactate-malate shuttle, $\alpha$-ketoglutarate dehydrogenase ( $a-\mathrm{KGDH})$, and mitochondrial respiratory chain complexes. Hence, the rate of energy production in cellular mitochondria will be compromised $[8,52,53]$.

Several investigations revealed that the administration of L-carnitine, acyl-L-carnitine, and creatine (mentioned as 'energy enhancing' compounds) improved the neurological functions in $\operatorname{HE}[54,55]$. It has also been reported that L-carnitine mitigated ammonia-induced CNS disorders in mice $[54,56]$. Acetyl-L-carnitine has also been shown to improve cerebral energy metabolism by activating mitochondrial enzymes of the electron transport chain [56]. Treatment combined with L-carnitine and acyl-L-carnitine also improved the cognitive functions and reduced behavioral abnormalities in patients with HE [56]. All these findings indicate the role of mitochondria and bioenergetic disturbances in the ammonia-induced CNS complications. The administration of other agents such as L-Ornithine-L-Aspartate also has been shown to affect blood and cerebrospinal fluid ammonia level [57]. Interestingly L-Ornithine-L-Aspartate elevated some amino acids in the CSF fluid, including taurine [57].

$\mathrm{mPT}$ plays a crucial role in the bioenergetics failure associated with $\mathrm{HE}$ and hyperammonemia. As cell volume regulation is an energy-dependent process, it is likely that mitochondrial dysfunction represents a critical factor in the development of brain edema in HE. Several studies indicate that taurine is localized in mitochondria [58-60]. Taurine also has been demonstrated to be a constituent of modified uridine residues in mitochondrial tRNA $[61,62]$. All these findings might indicate the importance of taurine in mitochondrial function, as well as its potential therapeutic applications in hyperammonemia.

It has been reported that taurine preserves $\mathrm{mPT}$ in cells [63]. Mitochondrial pH gradient and membrane potential are important factors for mitochondrial function $[14,29,30]$. Hence, chemicals that are able to localize in the mitochondrial matrix and regulate matrix $\mathrm{pH}$ are capable of preserving mitochondrial membrane potential. It has been found that taurine is involved in the mitochondrial matrix $\mathrm{pH}$ regulation $[14,29,30]$. The buffering property of taurine has also been found to be involved in the preservation of the function of mitochondrial matrix localized enzymes 
$[14,29,30]$. Interestingly, acyl-CoA dehydrogenase enzymes are demonstrated to have optimal activity in taurine buffer acyl-CoA dehydrogenase enzymes [14, $29,64]$. Thus, taurine prevents crucial enzyme defects in the hyperammonemic environment.

Brain edema is a major cause of mortality in hyperammonemia [65, 66]. Ammonia-induced brain edema has been directly related to astrocytes swelling [67]. Mechanisms that are involved in astrocyte swelling include oxidative and nitrosative stress, as well as mitochondrial dysfunction $[34,42,67]$. Ammonia has also caused mitochondrial permeability transition in cultured astrocytes [68]. It has been reported that taurine effectively mitigated astrocyte swelling in an in vitro system [69]. The effect of taurine on astrocyte swelling could be mediated by its positive effect on cell mitochondria.

Excess glutamic acid (Glu) in the ammonia-intoxicated brain is responsible for the over-activation of NMDA receptors and an excitotoxic response $[69,70]$. On the other hand, the anti-excitotoxic effect of taurine also has been shown in several investigations $[69,71$, 72]. Taurine might mitigate this response by activating inhibitory GABA receptors [69]. Hence, taurine might also mitigate the NMDA-mediated effects of ammonia in CNS.

In the current investigation, we found that taurine administration preserved the mitochondrial membrane potential and prevented mitochondrial swelling. These findings indicate that taurine is capable of protecting mitochondria as a crucial target of ammonia-induced toxicity in the brain and liver. The effect of taurine on mitochondrial respiratory chain complexes, as well as other enzymes affected by ammonia, requires further research.

We observed that taurine effectively ameliorated the direct effect of ammonia on isolated brain and liver mitochondria. This could make this amino acid a potential candidate against cerebral energy metabolism disturbances in acute and chronic liver injury and hepatic encephalopathy. Indeed, precise and in-depth in vivo and clinical evaluations will make clear the effect of taurine on mitochondria and reveal the therapeutic value of this amino acid against hyperammonemia complications.

\section{Acknowledgments}

The authors thank Pharmaceutical Sciences Research Center (PSRC) and the Vice-Chancellor of Research Affairs of Shiraz University of Medical Sciences for providing technical and financial support of the current investigation (Grant number 94-01-369741/9823).

\section{Disclosure}

Authors report no conflict of interest.

\section{References}

1. Felipo V. Hepatic encephalopathy: effects of liver failure on brain function. Nat Rev Neurosci 2013; 14: 851-858.

2. Shawcross D, Jalan R. The pathophysiologic basis of hepatic encephalopathy: central role for ammonia and inflammation. Cell Mol Life Sci 2005; 62: 2295-2304.

3. Norenberg M. Oxidative and nitrosative stress in ammonia neurotoxicity. Hepatology 2003; 37: 245-248.

4. Bosoi CR, Rose CF. Identifying the direct effects of ammonia on the brain. Metab Brain Dis 2008; 24: 95-102.

5. Albrecht J, Jones EA. Hepatic encephalopathy: molecular mechanisms underlying the clinical syndrome. J Neurol Sci 1999; 170: 138-146.

6. Wang Q, Wang Y, Yu Z, et al. Ammonia-induced energy disorders interfere with bilirubin metabolism in hepatocytes. Arch Biochem Biophys 2014; 555-556: 16-22.

7. Felipo V, Butterworth RF. Mitochondrial dysfunction in acute hyperammonemia. Neurochem Int 2002; 40: 487-491.

8. Ott P, Clemmesen O, Larsen FS. Cerebral metabolic disturbances in the brain during acute liver failure: From hyperammonemia to energy failure and proteolysis. Neurochem Int 2005; 47: 13-18.

9. O'Connor J-E, Costell M, Grisolía S. Protective effect of L-carnitine on hyperammonemia. FEBS Letter 1984; 166: 331-334.

10. Samuelsson C, Hillered L, Zetterling M, et al. Cerebral Glutamine and Glutamate Levels in Relation to Compromised Energy Metabolism: A Microdialysis Study in Subarachnoid Hemorrhage Patients. J Cereb Blood Flow Metab 2007; 27: 1309-1317.

11. Lai JCK, Cooper AJL. Neurotoxicity of ammonia and fatty acids: Differential inhibition of mitochondrial dehydrogenases by ammonia and fatty acyl coenzyme a derivatives. Neurochem Res 1991; 16: 795-803.

12. Bouckenooghe $T$, Remacle $C$, Reusens $B$. Is taurine a functional nutrient? Curr Opin Clin Nut Metab Care 2006; 9: 728-733.

13. Huxtable RJ. Physiological actions of taurine. Physiol Rev 1992; 72: 101-163.

14. Hansen SH, Andersen ML, Birkedal H, et al. The important role of taurine in oxidative metabolism. Adv Expt Med Biol 2006; 583: 129-135.

15. Oja SS, Saransaari P. Taurine as osmoregulator and neuromodulator in the brain. Metab Brain Dis 1996; 11: 153-164.

16. Schaffer S, Azuma J, Takahashi K, et al. Why is taurine cytoprotective? Adv Expt Med Biol 2003; 526: 307-321.

17. Heidari R, Babaei H, Eghbal MA. Cytoprotective effects of taurine against toxicity induced by isoniazid and hydrazine in isolated rat hepatocytes. Arh Hig Rada Toksikol 2013; 64: 15-24.

18. Sinha M, Manna P, Sil PC. Taurine, a conditionally essential amino acid, ameliorates arsenic-induced cytotoxicity in murine hepatocytes. Toxicol In Vitro 2007; 21: 1419-1428.

19. Ghosh M, Pal S, Sil PC. Taurine attenuates nano-copper-induced oxidative hepatic damage via mitochondria-dependent and NF- $\kappa B / T N F-\alpha$-mediated pathway. Toxicol Res 2014; 3: 474-486.

20. Schuller-Levis GB, Park E. Taurine: new implications for an old amino acid. FEMS Microbiol Lett 2003; 226: 195-202.

21. Menzie J, Pan C, Prentice H, et al. Taurine and central nervous system disorders. Amino Acids 2014; 46: 31-46.

22. Huxtable RJ. Taurine in the central nervous system and the mammalian actions of taurine. Prog Neurobiol 1989; 32: 471-533. 
23. Foos TM, Wu JY. The role of taurine in the central nervous system and the modulation of intracellular calcium homeostasis. Neurochem Res 2002; 27: 21-26.

24. Wu JY, Prentice H. Role of taurine in the central nervous system. J Biomed Sci 2010; 17: S1.

25. Saransaari P, Oja SS. Taurine and neural cell damage. Amino Acids 2000; 19: 509-526.

26. Zhou J, Li Y, Yan G, et al. Protective role of taurine against morphine-induced neurotoxicity in C6 cells via inhibition of oxidative stress. Neurotox Res 2011; 20: 334-342.

27. El Idrissi A. Taurine increases mitochondrial buffering of calcium: role in neuroprotection. Amino Acids 2008; 34: 321-328.

28. Hansen SH, Grunnet N. Taurine, Glutathione and Bioenergetics. Adv Expt Med Biol 2013; 776: 3-12.

29. Hansen SH, Andersen ML, Cornett C, et al. A role for taurine in mitochondrial function. J Biomed Sci 2010; 17: 1-8.

30. Jong CJ, Azuma J, Schaffer S. Mechanism underlying the antioxidant activity of taurine: prevention of mitochondrial oxidant production. Amino Acids 2011; 42: 2223-2232.

31. Heidari R, Jamshidzadeh A, Niknahad H, et al. Effect of taurine on chronic and acute liver injury: Focus on blood and brain ammonia. Toxicol Report 2016; 3: 870-879.

32. Caro AA, Adlong LW, Crocker SJ, et al. Effect of garlic-derived organosulfur compounds on mitochondrial function and integrity in isolated mouse liver mitochondria. Toxicol Lett 2012; 214: 166-174.

33. Zhao P, Kalhorn TF, Slattery JT. Selective mitochondrial glutathione depletion by ethanol enhances acetaminophen toxicity in rat liver. Hepatology 2002; 36: 326-335.

34. Felipo V. Advances in Cirrhosis, Hyperammonemia, and Hepatic Encephalopathy: Springer Science + Business Media, 2012.

35. Mosmann T. Rapid colorimetric assay for cellular growth and survival: application to proliferation and cytotoxicity assays. J Immunolog Method 1983; 65: 55-63.

36. Niknahad H, Heidari R, Alzuhairi AM, et al. Mitochondrial Dysfunction as a Mechanism for Pioglitazone-Induced Injury toward HepG2 Cell Line. Pharm Sci 2015; 20: 169-174.

37. Caro AA, Cederbaum AI. Synergistic toxicity of iron and arachidonic acid in HepG2 cells overexpressing CYP2E1. Mol Pharmacol 2001; 60: 742-752.

38. Held P. Luminescent Determination of ATP Concentrations Using the Clarity ${ }^{\mathrm{me}}$ Luminescence Microplate Reader. Nat Method, 2006

39. Bradford MM. A rapid and sensitive method for the quantitation of microgram quantities of protein utilizing the principle of protein-dye binding. Analyt Biochem 1976; 72: 248-254.

40. Hazell AS, Butterworth RF. Hepatic encephalopathy: An update of pathophysiologic mechanisms. Exp Biol Med 1999; 222: 99-112.

41. Lemberg A, Fernández MA. Hepatic encephalopathy, ammonia, glutamate, glutamine and oxidative stress. Ann Hepatol 2009; 8: 95-102.

42. Norenberg MD, Jayakumar AR, Rao KVR. Oxidative Stress in the Pathogenesis of Hepatic Encephalopathy. Metab Brain Dis 2004; 19: 313-329.

43. Wright CE, Tallan HH, Lin YY. Taurine: biological update. Annual Rev Biochem 1986; 55: 427-453.

44. Gürer H, Özgünes H, Saygin E, et al. Antioxidant Effect of Taurine Against Lead-Induced Oxidative Stress. Arch Envir Contam Toxicol 2014; 41: 397-402.

45. Das J, Ghosh J, Manna P, et al. Taurine protects rat testes against $\mathrm{NaAsO} 2$-induced oxidative stress and apoptosis via mitochondrial dependent and independent pathways. Toxicology Lett 2009; 187: 201-210.
46. Heidari R, Babaei H, Eghbal MA. Amodiaquine-induced toxicity in isolated rat hepatocytes and the cytoprotective effects of taurine and/or N-acetyl cysteine. Res Pharm Sci 2014; 9: 97-105.

47. Wu G, Yang J, Sun C, et al. Effect of taurine on alcoholic liver disease in rats. Amino Acids 2009; 36: 457-464.

48. Miyazaki T, Matsuzaki Y. Taurine and liver diseases: a focus on the heterogeneous protective properties of taurine. Amino Acids 2014; 46: 101-110.

49. Kobayashi T, Kuroda S, Tada M, et al. Calcium-induced mitochondrial swelling and cytochrome $\mathrm{c}$ release in the brain: its biochemical characteristics and implication in ischemic neuronal injury. Brain Res 2003; 960: 62-70.

50. Norenberg MD, Rao KVR, Jayakumar AR. Signaling factors in the mechanism of ammonia neurotoxicity. Metab Brain Dis 2008; 24: 103-117.

51. Palmi M, Youmbi GT, Fusi F, et al. Potentiation of mitochondrial $\mathrm{Ca} 2+$ sequestration by taurine. Biochem Pharmacol 1999; 58: 1123-1131.

52. Hertz L, Kala G. Energy metabolism in brain cells: effects of elevated ammonia concentrations. Metab Brain Dis 2007; 22: 199-218.

53. Ratnakumari L, Subbalakshmi GY, Murthy CRK. Acute effects of ammonia on the enzymes of citric acid cycle in rat brain. Neurochem Int 1986; 8: 115-120.

54. Malaguarnera M, Gargante MP, Cristaldi E, et al. Acetyl-L-carnitine treatment in minimal hepatic encephalopathy. Dig Dis Sci 2008; 53: 3018-3025.

55. Malaguarnera M. Effects of L-carnitine in patients with hepatic encephalopathy. W J Gastroenterol 2005; 11: 7197.

56. Malaguarnera M, Bella R, Vacante M, et al. Acetyl-l-carnitine reduces depression and improves quality of life in patients with minimal hepatic encephalopathy. Scand J Gastroenterol 2011; 46: 750-759.

57. Rose C, Michalak A, Rao KV, et al. L-Ornithine-L-aspartate lowers plasma and cerebrospinal fluid ammonia and prevents brain edema in rats with acute liver failure. Hepatology 1999; 30: 636-640.

58. Ottersen OP. Quantitative assessment of taurine-like immunoreactivity in different cell types and processes in rat cerebellum: an electronmicroscopic study based on a postembedding immunogold labelling procedure. Anatom Embryol 1988; 178: 407-421.

59. Bollard ME, Murray AJ, Clarke K, et al. A study of metabolic compartmentation in the rat heart and cardiac mitochondria using high-resolution magic angle spinning $1 \mathrm{H}$ NMR spectroscopy. FEBS Lett 2003; 553: 73-78.

60. Lobo MV, Alonso FJM, del Río RM. Immunocytochemical localization of taurine in different muscle cell types of the dog and rat. Histochem J 2000; 32: 53-61.

61. Suzuki T, Suzuki T, Wada T, et al. Taurine as a constituent of mitochondrial tRNAs: new insights into the functions of taurine and human mitochondrial diseases. EMBO J 2002; 21: 6581-6589.

62. Rubio MAT, Alfonzo JD. tRNA Modification, Editing, And Import In Mitochondria. In: Organelle Genetics. Springer-Verlag, Berlin Heidelberg 2012; 359-380.

63. Palmi M, Youmbi GT, Sgaragli G, et al. The mitochondrial permeability transition and taurine. Adv Exp Med Biol 2000; 483: 87-96.

64. Hansen SH, Birkedal H, Wibrand F, et al. Taurine and regulation of mitochondrial metabolism. Adv Exp Med Biol 2015; 803: 397-405.

65. Detry O, De Roover A, Honoré P, et al. Brain edema and intracranial hypertension in fulminant hepatic failure: pathophysiology and management. W J Gastroenterol 2006; 12: 7405-7412. 
66. Butterworth RF. Pathophysiology of hepatic encephalopathy: a new look at ammonia. Metab Brain Dis 2002; 17: 221-227.

67. Häussinger D, Schliess F. Pathogenetic mechanisms of hepatic encephalopathy. Gut 2008; 57: 1156-1165.

68. Norenberg MD, Baker L, Norenberg LO, et al. Ammonia-induced astrocyte swelling in primary culture. Neurochem Res 1991; 16: 833-836.

69. Zielińska M, Law RO, Albrecht J. Excitotoxic mechanism of cell swelling in rat cerebral cortical slices treated acutely with ammonia. Neurochem Int 2003; 43: 299-303.

70. Wejksza K, Rzeski W, Turski WA, et al. Ammonia at pathophysiologically relevant concentrations activates kynurenic acid synthesis in cultured astrocytes and neurons. Neurotoxicology 2006; 27: 619-622.

71. Wu ZY, Xu TL. Taurine-evoked chloride current and its potentiation by intracellular $\mathrm{Ca} 2+$ in immature rat hippocampal CA1 neurons. Amino Acids 2003; 24: 155-161.

72. Hilgier W, Oja SS, Saransaari P, et al. Taurine prevents ammonia-induced accumulation of cyclic GMP in rat striatum by interaction with GABAA and glycine receptors. Brain Res 2005; 1043: 242-246. 